\title{
Educação para a saúde no contexto online: relato de experiência
}

\author{
Health education in the online context: experience report \\ Educación sanitaria en el contexto online: informe de experiencia
}

Wemíria de Fátima Lima Lopes

ORCID: https://orcid.org/0000-0002-7444-2894 Universidade Federal do Recôncavo da Bahia, Brasil E-mail: wemiria.lopes@gmail.com

Isabella de Matos Mendes da Silva

ORCID: https://orcid.org/0000-0001-9700-0354 Universidade Federal do Recôncavo da Bahia, Brasil E-mail: isabellamatos@ufrb.edu.br

Fernando Vicentini

ORCID: https://orcid.org/0000-0002-0655-7374 Universidade Federal do Recôncavo da Bahia, Brasil E-mail: vicentini45@hotmail.com

Jorge Sadao Nihei

ORCID: https://orcid.org/0000-0003-2746-9987 Universidade Federal do Recôncavo da Bahia, Brasil E-mail: jsnihei@gmail.com

Gilyane Silva dos Santos

ORCID: https://orcid.org/0000-0003-3467-2190 Universidade Federal do Recôncavo da Bahia, Brasil E-mail: gilyanesilva@gmail.com

Juliel Conceição Barreto

ORCID: https://orcid.org/0000-0001-8231-1993 Universidade Federal do Recôncavo da Bahia, Brasil E-mail: julielbarreto14@gmail.com

Ana Lúcia Moreno Amor

ORCID: https://orcid.org/0000-0002-0977-1245

Universidade Federal do Recôncavo da Bahia, Brasil E-mail: ana_amor@ufrb.edu.br

\begin{abstract}
Resumo
A suspensão do ensino presencial pela pandemia do novo coronavírus em 2020 fez com que se buscassem metodologias aplicáveis à educação online. O podcast, por exemplo, é um arquivo digital de áudio transmitido por redes sociais para divulgar informações, constituindo-se em recurso educativo no processo ensino-aprendizagem. Este relato tem como objetivo apresentar o uso de metodologias no contexto da educação remota para graduandos da área da saúde. Trata-se de um estudo descritivo do tipo relato de experiência, a partir do olhar de docentes, discentes e da monitora no componente curricular Métodos de Diagnósticos Laboratoriais (MDL), no período de 14/09 a 19/12/2020, utilizando-se de momentos síncronos e assíncronos. Grupos com três ou quatro discentes elaboraram, ao longo do semestre, oito podcasts, um exemplo de procedimento operacional padrão (POP), dialogaram com docentes e monitora em ambientes virtuais e responderam sobre suas percepções quanto ao uso destas ferramentas para o processo ensino aprendizagem em um calendário acadêmico remoto. Destacam-se como respostas: $52,6 \%$ avaliaram as estratégias adotadas como muito boa e $42,1 \%$ como boa; $47,4 \%$ utilizaram o programa Anchor para gravação e edição do podcast e $73,7 \%$ não sentiram dificuldades quanto à gravação dos roteiros. Para o POP confeccionado, ficou o compromisso de realizá-lo em uma atividade extensionista, posteriormente. A busca pela melhor metodologia foi desafiadora. O podcast se mostrou uma metodologia satisfatória para o ensino da saúde no contexto da educação online para o componente curricular (MDL). Vislumbra-se socializar em comunidades diversas os podcasts produzidos, para popularizar os conhecimentos obtidos e suas relações.
\end{abstract}

Palavras-chave: Saúde coletiva; Educação em saúde; Educação médica.

\section{Abstract}

The suspension of classroom education due to the new coronavirus pandemic in 2020 led to the search for methodologies applicable to online education. Podcast, for example, is a digital audio file transmitted by social networks to disseminate information, constituting an educational resource in the teaching-learning process. This report aims to present the use of methodologies in the context of remote education for undergraduates in the health field. This is a descriptive study of the experience report type, from the perspective of teachers, students and monitors in the 
curricular component of Laboratory Diagnostic Methods (MDL), in the period from 14/09 to 19/12/2020, using synchronous and asynchronous moments. During the semester, groups with three or four students developed eight podcasts, an example of standard operating procedure (SOP), dialogued with teachers and monitors in virtual environments and answered their perceptions about the use of these tools for the teaching-learning process in a remote academic calendar. Stand out as answers: $52.6 \%$ evaluated the adopted strategies as very good and $42.1 \%$ as good; 47.4\% used the Anchor program to record and edit the podcast and $73.7 \%$ did not feel any difficulties regarding the recording of scripts. For the SOP made, it was committed to carry it out in an extension activity, later. The search for the best methodology was challenging. The podcast proved to be a satisfactory methodology for teaching health in the context of online education for the curricular component (CDM). It is envisaged to socialize the produced podcasts in different communities, in order to popularize the knowledge obtained and their relationships.

Keywords: Collective health; Health education; Medical education.

\section{Resumen}

La suspensión de la docencia en el aula por la nueva pandemia de coronavirus en 2020 llevó a la búsqueda de metodologías aplicables a la educación en línea. El podcast, por ejemplo, es un archivo de audio digital transmitido por las redes sociales para difundir información, constituyendo un recurso educativo en el proceso de enseñanzaaprendizaje. Este informe tiene como objetivo presentar el uso de metodologías en el contexto de la educación a distancia para estudiantes de pregrado en el campo de la salud. Se trata de un estudio descriptivo del tipo relato de experiencia, desde la perspectiva de docentes, alumnos y monitores en el componente curricular de Métodos de Diagnóstico de Laboratorio (MDL), en el período del 14/09 al 19/12/2020, utilizando síncronos y asincrónicos. momentos. Durante el semestre, grupos de tres o cuatro estudiantes desarrollaron ocho podcasts, ejemplo de procedimiento operativo estándar (POE), dialogaron con docentes y monitores en entornos virtuales y respondieron sus percepciones sobre el uso de estas herramientas para el proceso de enseñanza-aprendizaje en una Calendario académico remoto. Destacar como respuestas: 52,6\% evaluó las estrategias adoptadas como muy buenas y $42,1 \%$ como buenas; El 47,4\% utilizó el programa Anchor para grabar y editar el podcast y el 73,7\% no sintió ninguna dificultad en la grabación de guiones. Para el POE realizado, se comprometió a realizarlo en una actividad de extensión, posteriormente. La búsqueda de la mejor metodología fue un desafío. El podcast resultó ser una metodología satisfactoria para la enseñanza de la salud en el contexto de la educación en línea para el componente curricular (MDL). Se prevé socializar los podcasts producidos en diferentes comunidades, con el fin de popularizar los conocimientos obtenidos y sus relaciones.

Palabras clave: Salud colectiva; Educación para la salud; Educación médica.

\section{Introdução}

As metodologias ativas de aprendizagem consistem em alternativas para o ensino tradicional. Tais métodos de ensino visam tornar os discentes sujeitos ativos no processo de aprendizagem, de modo a promover uma práxis pedagógica capaz de desenvolver diferentes competências no ambiente de ensino (Pereira \& Santos Neto, 2020).

A internet surgiu na década de noventa no Brasil e, a partir daí, aconteceram mudanças significativas na forma de comunicação e a mesma é muito utilizada nos centros de pesquisa e nas instituições de ensino superior, sendo uma ferramenta fundamental no processo de educação e difusão do conhecimento (Abrantes \& Tavares, 2013). Salienta-se que esta ferramenta permite maior interação entre docentes e discentes, além de flexibilização dos estudos e estímulo à autonomia do estudante.

Para a formação profissional em saúde, iniciativas pedagógicas que sejam criativas, inovadoras e ousadas vão ao encontro da valorização das Tecnologias da Informação e Comunicação, uma vez que fortalecem a interface entre comunicação, ciência e sociedade (Curran et al., 2017). No âmbito internacional, já se reconhece a utilidade das mídias sociais como ferramentas para a promoção de projetos de pesquisa, fornecimento e disseminação de informações de saúde e facilitação da educação de estudantes e profissionais, a exemplo do Facebook, Twitter, Instagram e YouTube (Curran et al., 2017; Kelly et al., 2016; Pander, Pinilla, Dimitriadis, \& Fischer, 2014).

Nessa esteira, várias organizações internacionais e centros de pesquisa para a promoção de programas de ensino têm estimulado a adoção do espaço virtual e dos dispositivos móveis como recursos pedagógicos, reiterando a importância de um processo educacional que engloba as mídias digitais (França, Rabello \& Magnago, 2019).

Acompanhando a rápida tendência de expansão dessas tecnologias, percebe-se o surgimento e propagação de recursos voltados para o ensino virtual, com destaque para as plataformas online interativas, aplicativos para celulares e as redes sociais, 
que oferecem conteúdos bem acessíveis como videoaulas, sequências de imagens, livros digitais, roteiros de estudo, entre outros (Oliveira \& Martins, 2020).

Nesse sentido, desponta como uma das ferramentas de comunicação que tem se tornado cada vez mais popular o uso de podcast, uma mídia digital similar aos programas de rádio. Na realidade, o uso de podcast como ferramenta de ensinoaprendizagem iniciou a partir do rádio educativo brasileiro, que foi fundado concomitante com a primeira emissora em 1923. Em seguida, inúmeros projetos foram criados com a finalidade de levar informação às regiões distantes dos grandes centros, como alfabetização para adultos, capacitação para o trabalho, mobilização social e programas culturais. Para exemplificar, nos anos de 1994 e 2000, destacou-se o programa "Saúde no Ar", realizado pelo Ministério da Saúde, que tinha como objetivo oferecer conteúdos educativos dedicados a promover medidas preventivas de saúde junto à população de baixa renda (Bianco, 2019; Freire, 2013).

É nesse cenário que, quase um século após o advento do rádio educativo no Brasil, a ferramenta do podcast foi reinventada e atualmente permite aos ouvintes o acesso a conteúdos com um alto grau de pluralidade, que possibilitam uma ampliação temática da formação dos sujeitos, em virtude da possibilidade desses de tomarem contato com assuntos, posicionamentos e valores poucos discutidos tanto no rádio quanto em outros meios tradicionais (Freire, 2013).

O podcast é um arquivo digital de áudio transmitido pela internet para divulgar informações, constituindo-se em um recurso digital que pode ser utilizado como ferramenta educativa no processo de ensino-aprendizagem. Associados ao conceito de podcast estão: podcasting - refere-se ao ato de gravar ou divulgar os ficheiros na web; e, por fim, designa-se por podcaster - indivíduo que produz, ou seja, o autor que grava e desenvolve os ficheiros no formato áudio (Bottentuit \& Coutinho, 2007).

A utilização do podcast em educação pode trazer como vantagens: maior interesse na aprendizagem dos conteúdos devido a uma nova modalidade de ensino introduzida na sala de aula; recurso que ajuda nos diferentes ritmos de aprendizagem dos discentes, visto que os mesmos podem escutar inúmeras vezes um mesmo episódio a fim de melhor compreenderem o conteúdo abordado; possibilidade da aprendizagem dentro ou fora da escola (Bottentuit \& Coutinho, 2007).

Vale salientar que aprendizagem ativa ocorre quando o discente atua como protagonista em atividades interativas com outros discentes, aprendendo de maneira colaborativa. Nesse sentido, os trabalhos educativos com uso de podcast são geralmente realizados em grupos, momentos nos quais a aprendizagem colaborativa se sobrepõe à individualizada (Bottentuit \& Coutinho, 2007; Pereira \& Santos Neto, 2020). Devido a facilidade na comunicação, o acesso a informações em tempo hábil e a interação dinâmica e colaborativa que essa ferramenta possibilita no processo ensino-aprendizagem, o podcast permite que os discentes, por meio da troca entre pares e com os docentes, possam construir coletivamente conhecimentos sobre interesses em comum (Oliveira \& Martins, 2020).

O Procedimento Operacional Padrão (POP) é um documento/formulário onde se coloca a tarefa repetitiva de uma atividade prática (aulas laboratoriais e experimentos, por exemplo), na maneira e sequência que deve ser executada, o que dificulta os erros na execução desta. Nele contém: tarefa, executante, objetivo da tarefa, materiais necessários, processos, cuidados especiais, resultados esperados, ações corretivas e aprovação (Medeiros, 2010). A confecção e disponibilidade deste tipo de documento em aulas práticas efetiva-se como essencial para a organização e padronização das tarefas, constituindo-se em uma ferramenta que busca minimizar os erros na rotina de trabalho e/ou do laboratório.

Utilizar tecnologias/ferramentas diversas para a formação dos profissionais em saúde contribui para o desenvolvimento de recursos humanos qualificados para atender às necessidades de saúde da população, uma vez que promovem o desenvolvimento de competências técnicas, programáticas, gerenciais e administrativas aos futuros trabalhadores da saúde (Peres \& Ciampone, 2006).

Salienta-se que o uso de novas tecnologias tem ganhado força enquanto complemento às formas tradicionais de ensino presencial no ensino das ciências da saúde (Oliveira \& Martins, 2020). Entretanto, com a pandemia ocasionada pelo 
coronavírus da Síndrome Respiratória Aguda Grave 2 (SARS-CoV-2), detectado após a notificação de um surto em Wuhan, China, em dezembro de 2019 (Lana et al., 2020) e a suspensão do regime de aulas presenciais nas Instituições de Ensino Superior do país, particularmente na Universidade Federal do Recôncavo da Bahia (UFRB) (UFRB, 2020) no ano de 2020, docentes e discentes precisaram se adequar ao ensino online, a partir do uso de novas metodologias, bem como de modelos de avaliação alternativos. Considerando o exposto objetivou-se apresentar um relato de experiência acerca do uso destas metodologias de aprendizagem e avaliação no ensino da saúde no contexto da educação online que colaborasse no sucesso educativo dos sujeitos envolvidos.

\section{Metodologia}

Trata-se de um estudo descritivo, qualitativo, do tipo relato de experiência, com a finalidade de socializar sobre uso de metodologias de aprendizagem e avaliação no ensino da saúde no contexto da educação online no componente curricular CCS223 - Métodos Diagnósticos Laboratoriais (MDL) oferecido para discentes do Centro de Ciências da Saúde (CCS) / UFRB, especialmente para os estudantes dos cursos de Bacharelado Interdisciplinar em Saúde, Medicina, Enfermagem e Nutrição.

A suspensão do ensino presencial pela pandemia da doença do coronavírus 2019 (COVID-19) / Síndrome Respiratória Aguda Grave 2 (SARS-CoV-2), em 2020, fez com que se buscassem metodologias aplicáveis à educação online, constituindo-se como um desafio adaptar o formato do ensino presencial para o remoto. No que diz respeito ao referido componente, dispõe em sua ementa a avaliação dos métodos de diagnóstico das principais doenças infecciosas e parasitárias e autoimunes, bem como o estudo da correlação clínico laboratorial e epidemiológica dessas doenças; salientando-se seu objetivo principal que é descrever e analisar os principais diagnósticos microbiológicos, parasitológicos e/ou imunológicos e/ou imaginológicos e/ou moleculares em doenças infecciosas e parasitárias e suas aplicações.

O componente é trabalhado de forma presencial com 17 horas teóricas e 34 horas práticas por semestre letivo, contudo, considerando a situação da pandemia supracitada, para o semestre 2020.3, dentro de um calendário suplementar com ensino remoto, ele foi ofertado de forma online disposto em dois momentos: 13 horas de atividades síncronas e 38 horas de atividades assíncronas para o período de 14/09 a 19/12/2020.

Para as atividades síncronas (apresentando o tipo de método diagnóstico, seus princípios, indicação e análise dos resultados) foram utilizadas ferramentas que facilitam o aprendizado via ambiente virtual na plataforma Google meet a partir de uma exposição dialogada sobre os conteúdos abordados. Os temas trabalhados foram expostos com objetividade e clareza, articulando o conteúdo de maneira interdisciplinar com outros temas envolvidos sempre que possível. As atividade assíncronas (onde os discentes pesquisavam, em seu contexto social, casos clínicos/relatos, nos quais o método estudado era aplicado) foram conduzidas a partir de estudos que possibilitaram a confecção de materiais [relatos de casos via podcast e de Processo/Procedimento Operacional Padronizado/Padrão (POP)] relacionados com os métodos de diagnósticos apresentados (imunológico, parasitológico, imaginológico e molecular), bem como a realização de fóruns, videoaula, discussão de textos e exercícios diversos na turma virtual em plataforma institucional e em ambiente virtual do Google Sala de Aula.

Considerando os podcasts, orientações quanto a criação destes e os principais programas utilizados para produção e gravação, foram previamente repassadas aos discentes matriculados no componente MDL, bem como informações para a confecção do POP. Para o POP confeccionado, ficou o compromisso de realizá-lo em uma atividade extensionista, quando for possível o momento presencial.

Como critérios de avaliação foram utilizados os itens dispostos no Quadro 1: conteúdo; qualidade técnica; adaptação ao público; criatividade; tempo e cumprimento do prazo de entrega 
Quadro 1. Critérios em ficha de avaliação - Podcast e POP em MDL - Calendário suplementar, 2020.3.

1 - Conteúdo: objeto de estudo (conteúdo) está contextualizado. O tema está identificado dentro de um contexto social.

2 - Qualidade técnica: conhecimento, domínio e clareza do assunto, desenvolvimento sequencial da exposição, fluência na expressão verbal de ideias e questionamentos; coerência na forma de abordagem; os principais elementos do tema estão devidamente descritos e compatíveis para o recurso/ferramenta escolhida.

3 - Adaptação ao público: apresentação do tema clara, compatível, coerente e aplicável para o público.

4 - Criatividade (para o podcast) / Sequência lógica (para o POP): forma de apresentação, estética/artes, estrutura (sequência de apresentação)

5 - Tempo: entre 05 a 08 minutos (para o podcast)

6 - Cumprimento do prazo de entrega (material escrito/digitalizado e/ou gravado): reduzido em 10\% do valor a cada dia de atraso

Fonte: Os autores.

A inserção de uma discente como monitora nas atividades propostas, para o componente curricular em questão, favoreceu o acesso dos discentes a informações sobre novas ferramentas diagnósticas para doenças infecciosas e parasitárias, além do estímulo na busca de novos conhecimentos. Frequentemente os discentes eram incentivados pela monitora e docentes para interagir entre si e com estes por meio do fórum em plataforma digital. Durante o fórum, os discentes puderam debater sobre as lacunas na construção do podcast, POP e nos temas destes, buscando preenchê-las, a partir de referenciais diversos.

A percepção dos discentes quanto ao uso de podcast como ferramenta de aprendizagem foi avaliada por meio de um questionário semiestruturado disponibilizado por meio do Formulários Google, contendo 11 questões: (1) Como você avaliaria as estratégias adotadas no ensino de MDL neste modelo de ensino online? (2) Considerando que o componente possui carga horária de 3 horas semanais, que foram distribuídas em $2 \mathrm{~h}$ síncronas (1h aula e $1 \mathrm{~h}$ orientação) e $1 \mathrm{~h}$ assíncrona, qual o seu grau de satisfação quanto a carga horária das aulas síncronas? (3) Qual a sua opinião sobre as atividades dos podcasts como forma de construção e assimilação dos conhecimentos abordados no componente? (4) Você teve dificuldades quanto à elaboração dos textos/roteiros para os podcasts? (5) Você teve dificuldades quanto à gravação dos roteiros para os podcasts? (6) Qual o programa que você utilizou para gravar os podcasts? (7) Como você avaliaria o uso da plataforma Google Sala de Aula enquanto um espaço de apoio e disponibilização de materiais para a construção dos podcasts? (8) Como você avaliaria os materiais de apoio - vídeos, artigos, manuais, que foram disponibilizados por meio dessa plataforma? (9) Como você avaliaria a atuação dos docentes durante o semestre? (10) Como você avaliaria a atuação da monitora durante o semestre? (11) Quais as suas sugestões para a melhoria do componente? Foram utilizados os dados relacionados ao uso do podcast.

Este estudo integra ações educativas inseridas no projeto previamente aprovado pelo Comitê de Ética em Pesquisa com Seres Humanos sob o protocolo nº 023/2015, da Universidade Federal do Recôncavo da Bahia.

\section{Resultados}

Durante o semestre 2020.3, dezenove discentes dos cursos de Bacharelado Interdisciplinar em Saúde, Medicina, Enfermagem e Nutrição do CCS/UFRB cursaram o componente curricular optativo MDL. Especificamente para a produção da ferramenta podcast, estes discentes foram distribuídos em cinco trios e um quarteto, que confeccionaram, durante o semestre, oito produtos cada um, trabalhando os seguintes temas: 1. Interpretação Laboratorial do Hemograma; 2. Exames Parasitológicos de Fezes; 3. Análise Microbiológica de Água, Alimentos e Superfícies; 4. Imunodiagnósticos para Doenças Infecciosas e Parasitárias; 5. Análise Microbiológica de Amostras Clínicas e Antibiograma; 6. Diagnóstico Molecular de 
Agentes Infecciosos e Parasitários; 7. Métodos Laboratoriais de Identificação de Fungos; 8. Marcadores de Função Renal, Hepática e/ou Cardíaca em Doenças Infecciosas e Parasitárias.

Para a confecção do POP foi considerado apenas o tema "Análise Microbiológica de Água e/ou Alimentos". O principal intuito de se trabalhar com este recurso relaciona-se com o fato de que se constitui em uma metodologia auxiliar que serve de uma espécie de guia sobre como executar o método e/ou técnica, em questão. Por isso, quando possível realizá-lo em um momento presencial, a forma organizada como foi confeccionado poderá ajudar no sentido de dirigir a atividade prática de forma mais objetiva.

Sobre as metodologias trabalhadas, quando questionados como avaliaram as estratégias que foram adotadas para o ensino a distância do componente, $52,6 \%$ dos discentes avaliaram como muito boas, $42,1 \%$ avaliaram como boas e 5,3\% dos discentes avaliaram as estratégias de ensino como regulares (Figura 1A). Os resultados acerca da percepção dos discentes de MDL consideram o uso do podcast como uma ferramenta satisfatória.

No que diz respeito aos programas utilizados pelos discentes para gravação e edição dos podcasts: 47,4\% utilizaram o programa Anchor; 15,8\% fizeram uso do programa Audacity; 5,3\% utilizaram o programa Spreaker e 31,6\% fizeram uso de outros programas (Figura 1B). Quando se investigou sobre dificuldades com o uso dessa nova ferramenta quanto à gravação dos roteiros para os podcasts, $73,7 \%$ dos discentes referiram que não sentiram dificuldades.

As dificuldades mais relatadas foram:

Discente A - "Como cada um estava em sua casa, cada grupo tinha que mandar o seu áudio, alguns com qualidade ruim que para organizar foi um pouco complicado inicialmente, pois nunca tinha feito um podcast antes".

Discente B - "Por ser uma experiência nova, tive dificuldades com as primeiras gravações, ficava nervoso e errava algumas palavras, porém, a cada semana foi se tornando mais tranquilo".

Discente C - "Barulhos de fundo / barulhos externos, dificuldades para editar o áudio, para manusear as plataformas para a gravação dos podcast”.

Salienta-se que, para o podcast, como ponto positivo, as equipes trabalharam com atividades sequenciais, configurando-se como estratégias interessantes, como por exemplo, o ComSUS, sessão Descomplica e rádio MDL, procurando a partir das mesmas, o uso de linguagens simples, diretas e que pudessem dialogar, mesmo que virtualmente, com os ouvintes. Considerando se tratar de temática voltada para "Métodos de Diagnósticos Laboratoriais", maior ênfase foi dada para o método/técnica em questão.

Em relação às atividades de construção de podcast como forma de assimilação e sedimentação dos temas abordados, destacam-se as seguintes respostas:

Discente A - "Excelente forma de estudar e fazer uma apresentação visando entendimento do conteúdo por áudio, necessitando ser mais didático e treinar a oratória".

Discente B - "Considero uma estratégia produtiva que contribui muito no processo de assimilação dos conteúdos. A produção das atividades com antecedência garante maior aprimoramento e segurança na construção e sedimentação do aprendizado".

Discente $\mathbf{C}$ - "A ideia é bacana porque permite unir o conhecimento técnico da área com a ludicidade e saberes de outras esferas (como aplicativos para edição de áudio, etc)".

Discente D - "A proposta foi inovadora e realmente eficaz. Era necessário o estudo dos conteúdos todas as semanas". 
Discente E - "Muito bom. Utilizou a tecnologia para propor algo diferente, que não fosse tão "frio" como apenas um texto escrito".

Discente $\boldsymbol{F}$ - “Achei interessante, porém um pouco cansativo e mais trabalhoso do que só fazer um texto”.

Figura 1. Sobre as metodologias no componente CCS223 - Métodos de Diagnósticos Laboratoriais, 2020: (A) Avaliação das Estratégias de Ensino Adotadas. (B) Programas Utilizados para Gravação.
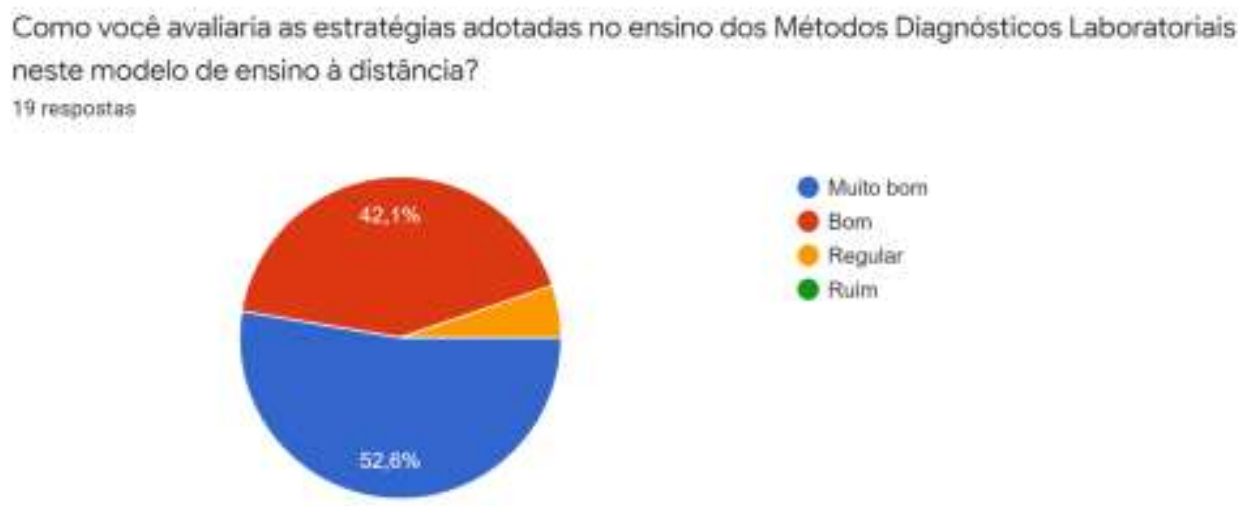

- Muito bom

$\mathbf{A}$

\section{Qual o programa que você utilizou para gravar os Podcasts?}

19 respostas
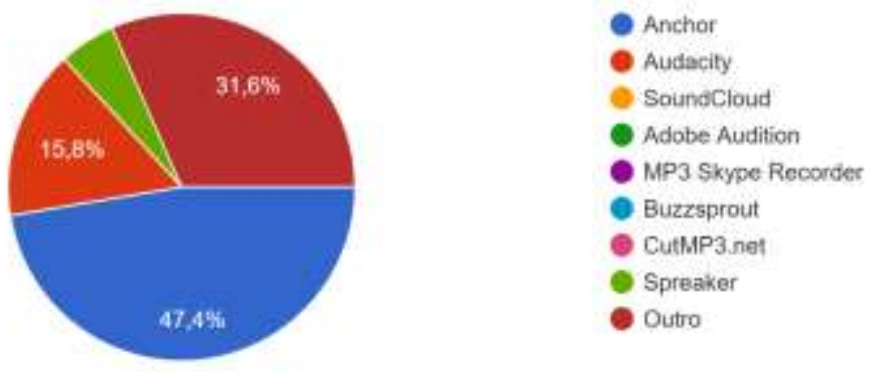

B

Fonte: Os autores (2020)

\section{Discussão}

Enfatiza-se que o componente MDL possui ênfase nas Análises Clínicas (Parasitologia, Imunologia, Microbiologia e Virologia), possibilitando ampliar os conhecimentos dos graduandos em saúde para temas essenciais na vida destes futuros profissionais da área. As infecções por parasitos entéricos e demais patógenos estão entre os mais frequentes agravos, estimando-se que, por exemplo, para as infecções intestinais o número de infectados no mundo seja de aproximadamente 3.5 bilhões de pessoas e no Brasil, variando por infecção, cerca de 130 milhões de habitantes são acometidos por alguma espécie de patógeno intestinal (Menezes, 2013). Desta forma, métodos laboratoriais para diagnóstico dos agentes etiológicos das doenças infectoparasitárias são de extrema relevância (Souza \& Amor, 2010). 
Conforme Jesus (2014), o podcast procura aliar tecnologia e oralidade, se mostra como uma linguagem oral dentro de um suporte digital, facilitando e ampliando a aproximação dos discentes a partir dos temas e histórias que são apresentados, características estas observadas no presente relato. E, segundo Pereira e Santos Neto (2020), apesar de não ser uma estratégia de ensino disseminada, os podcasts podem ser utilizados como uma ferramenta para revisar conteúdos abordados em sala de aula, apontando também para implantação deles como metodologia ativa no ensino superior.

As atividades de ensino a distância têm ganhado espaço progressivo na educação superior, buscando facilitar a comunicação do estudante com o docente. O ambiente virtual de aprendizagem, a exemplo do Google Sala de Aula, pode ser empregado como espaço de convergência, voltado para compartilhar as diversas vivências experimentadas pelos estudantes nos diferentes cenários, funcionando como uma excelente ferramenta para fins didático-pedagógico, quando utilizado de maneira planejada, pois possibilita diversificação metodológica; auxílio na organização de conteúdos e na interação entre os participantes (Bilthauer \& Gianotto, 2021).

Diante de um histórico de ensino médico tradicional, centrado no professor, pode haver resistência do uso de recursos didáticos modernos (Gorgens \& Andrade, 2018), a exemplo do podcast, como relatado pelo Discente F.

Como não há um modelo de ensino ideal e nem uma ferramenta que prometa resolver todos os problemas do ensino e da aprendizagem, o podcast deve ser entendido como mais uma ferramenta que pode ser utilizada em contexto pedagógico, possuindo atributos específicos e diferenciais que podem (e devem) ser combinados com outros métodos e com outras ferramentas em prol da melhoria da aprendizagem (Bottentuit \& Coutinho, 2007).

Resultados semelhantes ao deste relato foram obtidos com um grupo de discentes do $5^{\circ}$ período de Engenharia civil em uma instituição privada de ensino superior, em São João Del-Rei (Minas Gerais, Brasil), após experienciar o uso de podcasts enquanto ferramenta de ensino-aprendizagem. A maioria $(74,1 \%)$ afirmou que recomendaria a gravação de podcasts como atividade de ensino para outros discentes, $3,7 \%$ talvez recomendaria e $22,2 \%$ não recomendaria. A autonomia discente, estimulada durante a formação dos grupos, a escolha do referencial bibliográfico, como elaborar o roteiro, escolha dos papéis de cada integrante nos episódios, a dedicação em produzir um conteúdo informativo, buscando diferentes referências, o maior engajamento, contribuem para o aprendizado ativo, auto-orientação e desenvolvimento de habilidades de trabalho em equipe (Pereira \& Santos Neto, 2020).

Vale ressaltar que as gravações não necessitam de recursos avançados para ficarem numa qualidade satisfatória, basta que sejam gravadas em ambientes com pouco ruído. Podem ainda ser combinadas com banda sonora de fundo, o que, em alguns casos, poderá agregar mais valor aos episódios, tornando-os mais atrativos e contextualizados (Bottentuit \& Coutinho, 2007).

Salienta-se que, como qualquer elaboração tecnológica, a confecção de um podcast demanda esforços de produção, de modo que o resultado final da realização estará relacionado com o teor de seu conteúdo e com a forma de gravação e edição do material. O pouco domínio com os recursos de gravação e edição, a indisponibilidade de equipamentos, a falta de tempo para se dedicar à edição, em que a cada minuto apresentado, é necessário cerca de quatro vezes mais tempo para edição e gravação, somado a ambientes ruidosos que não favorecem as gravações, são os principais obstáculos referenciados no primeiro contato com a ferramenta de podcast dentro do cenário escolar, e, também citados por alguns discentes deste estudo. Tais considerações não diminuem a importância dos aspectos produtivos na elaboração de podcasts voltados a objetivos educativos. Além disso, à medida que gradualmente são inseridos músicas, vinhetas, efeitos sonoros, divisão em blocos, seleção das seções mais interessantes, o programa se torna mais dinâmico, potencializa o desenvolvimento da empatia dos sujeitos com sua produção e os estimula a se dedicar e aprofundar os conhecimentos técnicos a partir do uso de podcast (Freire, 2013). Ressaltase que os discentes estimulados a gravar episódios podem aprender mais, pois terão maior preocupação em preparar um bom 
texto e disponibilizar um material correto e coerente para divulgação, haja vista que o ato de falar e ouvir constitui uma atividade de aprendizagem mais significativa do que somente o ato de ler (Bottentuit \& Coutinho, 2007).

Sabe-se que os discentes de hoje não são os mesmos para os quais o sistema educacional foi inicialmente elaborado e representam gerações que cresceram em contato com novas tecnologias. A prática pedagógica universitária não se restringe a informar o discente, pois existem vários espaços fora da universidade onde podem ser encontradas informações (Gorgens \& Andrade, 2018). Assim, para aprender a aprender, identificando conhecimentos prévios e formulando questões para a busca de respostas cientificamente consolidadas, conforme as Diretrizes Curriculares Nacionais dos Curso de Medicina (Brasil, 2014) e de outros cursos da área da saúde, é necessário manuseio dos recursos tecnológicos existentes em favor de sua formação e atualização (Gorgens \& Andrade, 2018).

A transição do ensino presencial para o remoto exigiu adaptações e flexibilidade, e tais mudanças transformaram o cenário do processo de ensino e aprendizagem, apontando que a tecnologia pode ser ainda mais explorada, tendo perspectivas permanentes (Santos, Nery, Carvalho, \& Cecilio-Fernandes, 2021). Recursos digitais potencializam a dinamização do desenvolvimento de ações de educação na saúde (França, Rabello \& Magnago, 2019). As Instituições de Ensino Superior (IES) têm sido estimuladas a refletir acerca das mudanças do processo da educação e romper com estruturas cristalizadas de ensino tradicional. Os métodos inovadores de ensino e aprendizagem mostram claramente o movimento de migração do "ensinar" para o "aprender", o desvio do foco do docente para o discente, que assume a corresponsabilidade pelo seu aprendizado.

Um dos grandes desafios para as IES está na formação contínua do corpo docente, proporcionando infraestrutura, quando necessária, para a utilização dos variados métodos de ensino e aprendizagem (Souza, Iglesias \& Pazin-Filho, 2014). Hoje é difícil imaginar um curso superior que não utilize computador ou internet em algum momento do curso. À medida que as tecnologias se aprimoram, as possibilidades se tornam mais atrativas e mais complexas, ampliando, inclusive, o conceito de estar junto no tempo e no espaço (Gorgens \& Andrade, 2018). Por isso, o docente precisa reconhecer a tecnologia como recurso, atuando como mediador na construção do conhecimento (Pereira, Areco, Tarcia \& Sigulem, 2016).

\section{Conclusão}

A pandemia da COVID-19 e a consequente necessidade de suspensão das aulas presenciais e a adoção do ensino online exigiram de docentes e discentes uma nova postura, moldada por novos conhecimentos e habilidades, como por exemplo, a habilidade de manusear recursos tecnológicos existentes em favor de sua formação e atualização, trazendo com isso cada vez mais possibilidades didáticas. O uso do podcast (especialmente) e do POP, se constituiram, para este componente curricular, em metodologias que trabalharam a construção de conhecimentos a partir da vivência de experiências coletadas pelos discentes, que puderam interagir a partir da construção dos produtos. Particularmente, o recurso do podcast permitiu exercitar uma forma diferente de comunicação, apreender o conteúdo e identificar o uso dos principais métodos diagnósticos de doenças infecciosas e parasitárias, a partir de experiências de familiares e amigos. Desta forma, cada tema foi explorado ao longo dos episódios, o que favoreceu o desenvolvimento da autonomia. Considerando o relato dos discentes do componente curricular MDL, o uso de podcast se mostrou uma metodologia ativa satisfatória para o ensino da saúde no contexto da educação online, facilitando o processo ensino-aprendizagem.

O uso de ferramentas digitais como tecnologias da informação e comunicação, a exemplo da confecção e socialização do podcast permite também contribuir para o desenvolvimento acadêmico da atenção primária à saúde, com ênfase na medicina de família e comunidade, bem como para outros profissionais da atenção primária à saúde. Considerando o tema em destaque "diagnósticos laboratoriais", este poderá abordar adequadamente questões relevantes para a medicina e saúde, permitindo orientar, informar de forma mais coloquial ou não, a depender do público de ação, colaborando na construção do 
conhecimento deste público. Se configurando como uma medida profilática, no caso do diagnóstico de doenças infecciosas e parasitárias e de identificação e controle de doenças não transmissíveis, como forma de popularizar o conhecimento acerca dos métodos diagnósticos laboratoriais.

\section{Agradecimentos}

Agradecimento pelo apoio financeiro da Pró-Reitoria de Graduação (PROGRAD) da UFRB para a bolsa acadêmica da monitora que permitiu à discente apoiar e auxiliar nas demandas do componente CCS223 - Métodos de Diagnósticos Laboratoriais.

\section{Referências}

Abrantes M. M., \& Tavares E. C. (2013). Usando a Internet para atualização em Pediatria. In: Leão et al. Pediatria Ambulatorial. Belo Horizonte: Coopmed, 1423-29.

Bianco, N. R. D. (2009). Aprendizagem por rádio. In: Litto, M., \& Formiga, M. M. Educação à Distância: o estado da arte. São Paulo: Pearson Education do Brasil.

Bilthauer, M. I., \& Gianotto, D. E. P. (2021). Contribuições, potencialidades e dificuldades do ambiente Google Sala de Aula para o processo ensino e aprendizagem. Research, Society and Development, [Sl.], 10(8), p. e1710817097, 2021. 10.33448/rsd-v10i8.17097.

Bottentuit, J. B., \& Coutinho, C. P. (2007). Podcast em educação: um contributo para o estado da arte. In: IX Congresso Internacional Galego-Portugués de Psicopedagoxía. p.78. http://repositorium.sdum.uminho.pt/bitstream./1822/7094/1/pod.pdf.

Brasil (2014). Ministério da Educação. Conselho Nacional da Educação. Resolução No 3, de 20 de junho de 2014. Institui Diretrizes Curriculares Nacionais do Curso de Graduação em Medicina e dá outras providências. Brasília: Ministério da Educação.

Curran, V., Matthews, L., Fleet, L., Simmons, K., Gustafson, D. L., \& Wetsch, L. (2017). A Review of Digital, Social, and Mobile Technologies in Health Professional Education. J Contin Educ Health Prof. [internet]. 37(3):195-206. http://dx.doi.org/10.1097/CEH.0000000000000168.

França, T., Rabello, E. T., \& Magnago, C. (2019). As mídias e as plataformas digitais no campo da Educação Permanente em Saúde: debates e propostas. Saúde em Debate. Rio de Janeiro, 43(Especial 1):106-115. http://dx.doi.org/10.1590/0103-11042019s109.

Freire, E. P. A. (2013) Podcast na educação brasileira: natureza, potencialidades e implicações de uma tecnologia da comunicação. 338 f. Tese (Doutorado em Educação) - Universidade Federal do Rio Grande do Norte, Natal. http://repositorio.ufrn.br/jspui/handle/123456789/14448.

Gorgens, P. R. C., Andrade, P. C. R. (2018). Educação médica e tecnologias digitais de informação e comunicação: possibilidades e dilemas. Rev Med Minas Gerais, 28: e-2004. http://www.dx.doi.org/10.5935/2238-3182.20180154.

Jesus, W. B. (2014). Podcast e educação: um estudo de caso. 56 f. Dissertação (Mestrado) - Universidade Estadual Paulista, Instituto de Biociências de Rio Claro. http://hdl.handle.net/11449/121992.

Kelly, B. S., Redmond, C. E., Nason, G. J., Healy, G. M., Horgan, N. A, \& Heffernan, E. J. (2016). The use of Twitter by Radiology Journals: An Analysis of Twitter Activity and Impact Factor. J AM Coll Radiol [internet]. 13(11):1391-1396. https://doi.org/10.1016/j.jacr.2016.06.041.

Lana, R. M., Coelho, F. C., Gomes, M. F. C., Cruz, O. G., Bastos, L. S., Villela, D. A. M. et al. (2020). Emergência do novo coronavírus (SARS-CoV-2) e o papel de uma vigilância nacional em saúde oportuna e efetiva. Cad. Saúde Pública 36(3):e00019620. http://www.dx.doi.org/10.1590/0102-311X00019620.

Medeiros, T. B. (2010). POP - Procedimento Operacional Padrão. Trabalho de Conclusão de Curso. Fundação Educacional do Municipio de Assis - FEMA Assis, São Paulo. 56p.

Menezes, R. A. O. (2013). Caracterização epidemiológica das enteroparasitoses evidenciadas na população atendida na Unidade Básica de Saúde Congós no município de Macapá-Amapá. 158 f. Dissertação (Mestrado em Ciências da Saúde) - Departamento de Pós-Graduação, Universidade Federal do Amapá, Macapá. http://repositorio.unifap.br:80/jspui/handle/123456789/191.

Oliveira, F. J. M., \& Martins, M. A. (2020) Plataformas Digitais no Ensino de Pediatria da UFMG. Rev Med Minas Gerais. 30:e-30501. http://www.dx.doi.org/10.5935/2238-3182.20200067.

Pander, T., Pinilla, S., Dimitriadis, K., \& Fischer, M. R. (2014). The use of Facebook in medical education - A literature review. GMS Z Med Ausbild [internet]. 31(3):Doc33. https://doi.org/10.3205/zma000925.

Pereira, A. R., \& Santos Neto, F. A. (2020). Podcast como estratégia de aprendizagem no ensino superior. Pensar Acadêmico, Manhuaçu. 18(4):769-782. http://dx.doi.org/10.21576/pa.2020v18i4.1941.

Pereira, T. A., Areco, K. C. N., Tarcia, R. M. L., \& Sigulem, D. (2016) Uso das Tecnologias de Informação e Comunicação por Professores da Área da Saúde da Universidade Federal de São Paulo. Rev Bras Educ Med. 40(1):59-66. https://doi.org/10.1590/1981-52712015v40n1e01482015.

Peres, A. M., \& Ciampone, M. H. T. (2006). Gerência e competências gerais do enfermeiro. Texto Contexto Enferm, Florianópolis, 2006 Jul-Set; 15(3): 492-9. 
Research, Society and Development, v. 10, n. 11, e224101119669, 2021

(CC BY 4.0) | ISSN 2525-3409 | DOI: http://dx.doi.org/10.33448/rsd-v10i11.19669

Santos, L. L., Nery, N. D. M. L., Carvalho, E. R., \& Cecilio-Fernandes D. (2021). Transição do ensino presencial para o remoto em tempos de COVID-19: Perspectiva docente. Scientia Medica, 2021; 31(1), e39547. https://doi.org/10.15448/1980-6108.2021.1.39547.

Souza, C. S., Iglesias, A. G., \& Pazin-Filho, A. (2014). Estratégias inovadoras para métodos de ensino - Aspectos Gerais. Medicina (Ribeirão Preto). 47(3):284-92. https://www.revistas.usp.br/rmrp/article/view/86617.

Souza, R. F., \& Amor, A. L. M. (2010). Controle de qualidade de técnicas realizadas nos laboratórios de parasitologia da Secretaria Municipal de Saúde do Município de Salvador, Bahia. Revista Brasileira de Análises Clínicas, 42:101-106. https://xdocs.com.br/doc/artigo-controle-qualidade-parasitologiawvo90241xmoj.

Ufrb (2020). Portaria n. 322, de 17/03/2020, da Reitoria da Universidade Federal do Recôncavo da Bahia. Boletim de Pessoal - Ano XIV - No 049/2020. file:///C:/Users/Administrador/Downloads/BP_49.-2020\%20(1).pdf. 